\title{
Deep modulation of second-harmonic light by wavelength detuning of a laser diode
}

Christensen, Mathias; Hansen, Anders Kragh; Noordegraaf, Danny; Jensen, Ole Bjarlin; Skovgaard, Peter M. W.

\section{Published in:}

Applied Optics

Link to article, DOI:

10.1364/AO.56.002250

Publication date:

2017

Document Version

Peer reviewed version

Link back to DTU Orbit

Citation (APA):

Christensen, M., Hansen, A. K., Noordegraaf, D., Jensen, O. B., \& Skovgaard, P. M. W. (2017). Deep

modulation of second-harmonic light by wavelength detuning of a laser diode. Applied Optics, 56(8), 2250-2254.

https://doi.org/10.1364/AO.56.002250

\section{General rights}

Copyright and moral rights for the publications made accessible in the public portal are retained by the authors and/or other copyright owners and it is a condition of accessing publications that users recognise and abide by the legal requirements associated with these rights.

- Users may download and print one copy of any publication from the public portal for the purpose of private study or research.

- You may not further distribute the material or use it for any profit-making activity or commercial gain

- You may freely distribute the URL identifying the publication in the public portal

If you believe that this document breaches copyright please contact us providing details, and we will remove access to the work immediately and investigate your claim 


\title{
Deep Modulation of Second Harmonic Light by Wavelength Detuning of a Laser Diode
}

\author{
Mathias Christensen, $, 1,2,{ }^{*}$ Anders K. Hansen, ${ }^{2}$ Danny NoordegraAf, ${ }^{1}$ Ole B. \\ Jensen, ${ }^{2}$ Peter M. W. SkovgaArd ${ }^{1}$ \\ ${ }^{1}$ Norlase ApS, Risø Campus, Frederiksborgvej 399, 4000 Roskilde, Denmark \\ ${ }^{2}$ Technical University of Denmark, Department of Photonics Engineering, Frederiksborgvej 399, 4000 Roskilde, Denmark \\ *Corresponding author: mac@norlase.com
}

Received XX Month XXXX; revised XX Month, XXXX; accepted XX Month XXXX; posted XX Month XXXX (Doc. ID XXXXX); published XX Month XXXX

\begin{abstract}
Power modulated visible lasers are interesting for a number of applications within areas such as laser displays and medical laser treatments. In this paper, we present a system for modulating the second harmonic (SH) light generated by single-pass frequency doubling of a distributed feedback (DFB) master oscillator power amplifier (MOPA) laser diode with separate electrical contacts for the MO and the PA. A modulation depth in excess of $\mathbf{9 7 \%}$ from $0.1 \mathrm{~Hz}$ to $10 \mathrm{kHz}$ is demonstrated. This is done by wavelength tuning of the laser diode using only a $40 \mathrm{~mA}$ adjustment of the current through the MO. The bandwidth of the modulation is limited by the electronics. This method has the potential to decrease the size as well as cost of modulated visible lasers. The achievable optical powers will increase as DFB MOPAs are further developed.
\end{abstract}

OCIS codes: (140.3490) Lasers, distributed-feedback; (140.3515) Lasers, frequency doubled; (140.3480) Lasers, diode pumped; (140.7300) Visible lasers; (140.3538) Lasers, pulsed.

http://dx.doi.org/10.1364/AO.99.099999

\section{Introduction}

A great deal of research has gone into developing green lasers with the capability of arbitrary modulation of the output power, as these lasers are interesting for laser displays[1] and medical applications[2]. Within the field of ophthalmology, doctors require watt-level power, often in the visible wavelength range, with close to $100 \%$ modulation depth. Currently pulse lengths in excess of $50 \mathrm{~ms}$ are the most widely used[3], but recent research has shown that treatment with $\mu$ s pulses might decrease the damage of the retina and increase patient comfort without compromising the treatment results[4].

Laser diodes are ideal light sources for power modulation due to their fast response to current changes. This is utilized extensively in the telecom industry where laser diodes modulated at $\mathrm{GHz}$ frequencies are a commodity today. However, these laser diodes are infrared and low power.

Frequency doubled diode pumped solid state (DPSS) lasers have produced $1.6 \mathrm{kHz}$ modulation with $300 \mathrm{~mW}$ output power[5]. However, DPSS lasers are limited in their modulation bandwidth by the upper state lifetime of the solid state laser crystals which is typically $100-300$ $\mu s[6]$ and to our knowledge a DPSS laser with the combination of wattlevel powers and $\mu$ s pulses without external modulation of the power has not been demonstrated. Optically pumped semiconductor (OPS) lasers, on the other hand, can generate multiple watts of powers in combination with short pulse durations[7]. Both DPSS and OPS lasers are however, quite bulky, expensive and their wall-plug efficiency is low.
Ideally, one would like to use laser diodes emitting directly in the green or yellow. However, since high power laser diodes in this spectral region are not yetavailable, an attractive alternative is second harmonic generation (SHG) using near infrared (NIR) laser diodes. Previously, 3.7 $\mathrm{W}$ of green light by frequency doubling of $9.5 \mathrm{~W}$ NIR light from a distributed Bragg reflector (DBR) tapered laser diode in a cascaded single-pass configuration was demonstrated[8]. In principle the $\mathrm{SH}$ output of such a system can be modulated by gain-switching, i.e. turning the laser diode on and off. However, this yields two main problems: First of all the temperature and therefore wavelength of the laser diode will fluctuate on time scales from milliseconds to seconds. This makes it difficult to obtain stable modulation of the SH light because of the narrow spectral acceptance bandwidth of the non-linear crystal[9]. Secondly the current needed to obtain multiple watts of visible power from such a system is on the order of $10 \mathrm{~A}[8]$, which means on-off modulation is limited in bandwidth and requires expensive drivers.

One way around these issues is to take advantage of the narrow spectral acceptance bandwidth of the crystal to modulate the SH power by detuning the wavelength of the laser diode away from phase matching. The modulation scheme has previously been used to obtain MHz modulation of blue light for optical storage[10] and green light for laser displays[11]. In both cases a DBR laser and nonlinear crystals with channel waveguides were used, since these enable very high conversion efficiency[12]. SHG systems using channel waveguides in nonlinear crystals cannot be scaled to the multiple watt-level powers needed for medical treatment [13]. A 90\% modulation depth of $1.5 \mathrm{~W}$ SH light has 
been achieved using wavelength detuning of DBR tapered laser diodes[14]. In this work, however, the modulation depth was limited by the mode hops of the laser. High modulation depth of DBR tapered lasers with a detuning scheme has so far only been obtained at very high diode temperature $\left(50^{\circ} \mathrm{C}\right)[15]$.

In this paper we present $>97 \%$ modulation depth of the SH light using wavelength detuning of a distributed feedback (DFB) master oscillator power amplifier (MOPA) laser diode. Furthermore, we explore the mode structure of the DFB MOPA and present how this structure is beneficial for the modulation scheme.

\section{Setup and Results}

The setup is sketched in Fig. 1. The laser diode was a $1064 \mathrm{~nm}$ monolithic DFB MOPA laser diode from QPC Lasers, similar to that described in[16]. In these laser diodes the master oscillator (MO) consists of a DFB ridge waveguide section from which the output is allowed to diverge through a tapered power amplifier (PA). The whole structure is mounted p-side up to allow for separate contacts for the MO and PA. The output power of the laser diode was $3 \mathrm{~W}$ with $150 \mathrm{~mA}$ through the MO, 4 A through the PAand the laser temperature stabilized at $20^{\circ} \mathrm{C}$. The laser diode was collimated and then the beam was passed through a $30 \mathrm{~dB}$ optical isolator to protect the diode from back reflections. After the collimation the laser diode had an $\mathrm{M}^{2}$, measured using the second moment width, of 2.8 in the horizontal direction and 1.9 in the vertical direction. A small portion of the light was picked out using a polarizing beam splitter (PBS) and half of it was focused onto a $350 \mathrm{MHz}$ photodiode to monitor the NIR light during modulation and the second half was coupled into a scanning Fabry-Pérot Interferometer (FPI). The scanning FPI was used to monitor the laser linewidth, ensuring that the laser diode was not affected by external optical feedback. A second $30 \mathrm{~dB}$ optical isolator provided additional protection from optical feedback and the polarization of the NIR light was adjusted using a half-wave plate. The NIR light was focused to a $1 / \mathrm{e}^{2}$ diameter of approximately $60 \mu \mathrm{m}$ in a $50 \mathrm{~mm}$ long bulk periodically poled magnesium oxide doped lithium niobate (PPLN) crystal using two plane folding mirrors and a $100 \mathrm{~mm}$ focal length lens, generating green $\mathrm{SH}$ light at $532 \mathrm{~nm}$. After the crystal, the remaining NIR light was filtered out using a dichroic mirror and the SH light was refocused onto another $350 \mathrm{MHz}$ photodiode. The light was focused onto both photodiodes to avoid errors in the measurement from pointing instabilities or spatial mode changes. A thermal power meter was used for calibrating the fast photodiode. The voltage over the MO and the response from the two $350 \mathrm{MHz}$ photodiodes were monitored on a $1 \mathrm{GHz}$ oscilloscope.

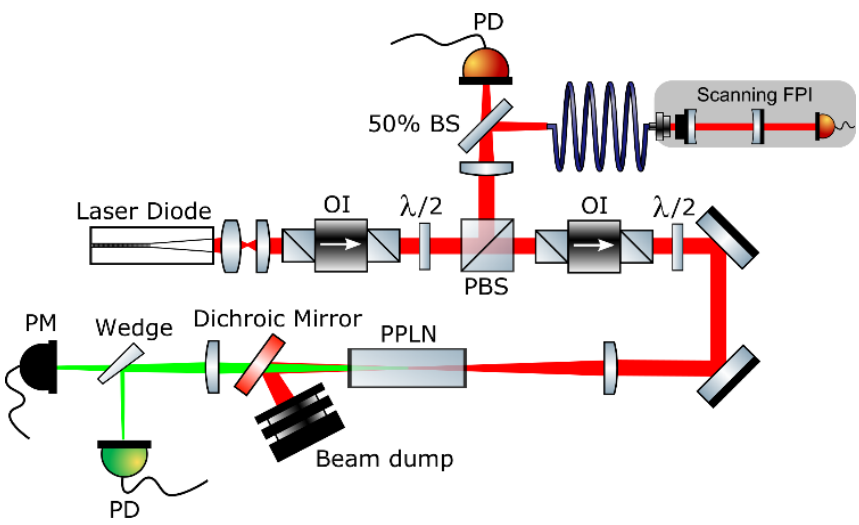

Fig. 1. Sketch of the setup. PD: Photodiode. OI: Optical isolator. $\lambda / 2$ : Halfwave plate. BS: Beam splitter. PBS: Polarizing beam splitter. FPI: FabryPérot interferometer. PPLN: Periodically poled lithium niobate crystal. PM: Thermal power meter.
The laser diode has some important mode characteristics which must be understood to obtain stable modulation by wavelength detuning. Fig. 2 shows how the peak wavelength of the laser changes when the current through the MO is changed. An important feature here is that laser diodes based on DBR and DFB technology have different mode hop behaviors. For a DFB laser the mode hops result in an increase of the wavelength as the current is increased, whereas the wavelength for a DBR laser would decrease. This is advantageous for modulation because a single mode hop for a DFB laser diode will shift the wavelength far from phase-matching. Furthermore, the mode hop positions of the DFB laser diode are independent of the direction that the current is scanned, i.e., there is no mode hysteresis. The lack of mode hysteresis in the DFB laser diode is another importantadvantage for the wavelength detuning modulation scheme, since it results in good reproducibility of the pulses. The gray background indicates regions of spectrally multimode operation, with greatly reduced frequency doubling efficiency. To avoid damage to the laser diode the DFB had to be operated between $100 \mathrm{~mA}$ and $150 \mathrm{~mA}$, and for this reason the modulation was performed by switching between 100 and $140 \mathrm{~mA}$.

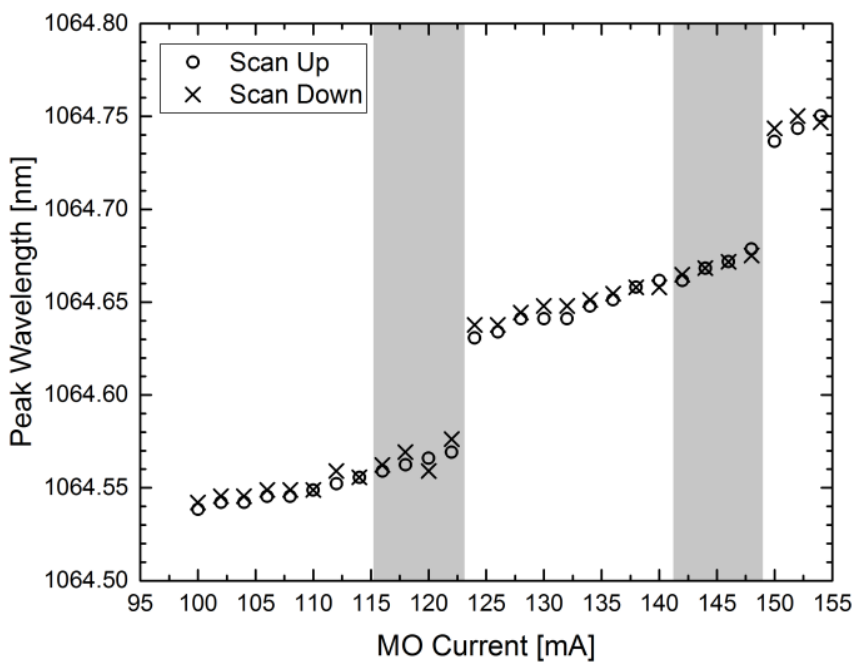

Fig. 2. Wavelength of the laser diode versus the current through the MO. The current through the PA was kept at $4 \mathrm{~A}$. The data were acquired by first scanning up in steps of $2 \mathrm{~mA}$ and then back down. Gray background indicates areas of spectral multimode operation. The data were obtained with the laser diode in CW operation.

Fig. 3 shows the spectrum of the laser diode at MO currents of $100 \mathrm{~mA}$ and $140 \mathrm{~mA}$, measured with an optical spectrum analyzer (OSA). The full width half maximum (FWHM) linewidth of the laser was below 5 pm (limited by the OSA resolution) at both points. The side modes at roughly $-20 \mathrm{dBc}$ are artifacts from the OSA. The change in wavelength for these two outer points of the modulation is $\Delta \lambda=120 \mathrm{pm}$. 


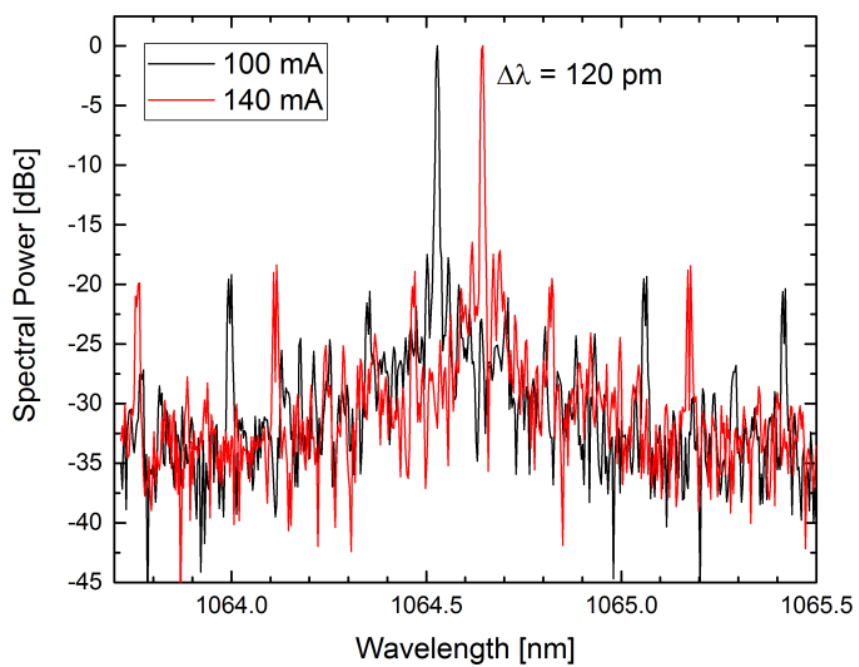

Fig. 3. Spectrum of the laser diode at 100 and $140 \mathrm{~mA}$, with the laser diode in $\mathrm{CW}$ operation. The side modes at -17 to $-20 \mathrm{dBc}$ are measurement artifacts from the OSA. The PA current was 4A.

The acceptance bandwidth of the crystal was measured to be $59 \mathrm{pm}$ (FWHM), i.e. significantly lower than the wavelength shift of the laser diode. The acceptance bandwidth is shown in Fig. 4 together with the wavelength shift of the laser diode to show that the modulation shifts the wavelength outside the peak of the conversion efficiency. As expected, the acceptance bandwidth is larger than the theoretical value for a $50 \mathrm{~mm}$ PPLN crystal of $42 \mathrm{pm}$. This is mainly due to the use of a focused beam instead of plane waves and crystal poling imperfections. The acceptance bandwidth has been shown to increase slightly for increasing power levels[17]. However, the increase was only $20 \%$ when going from $1 \mathrm{~W}$ input power to $8.5 \mathrm{~W}$ and the broadening was asymmetrical, mainly affecting the high wavelength drop-off of the efficiency. Itshould, therefore, be straight forward to scale the SH power without affecting the modulation depth, once higher power DFB MOPA laser diodes become available.

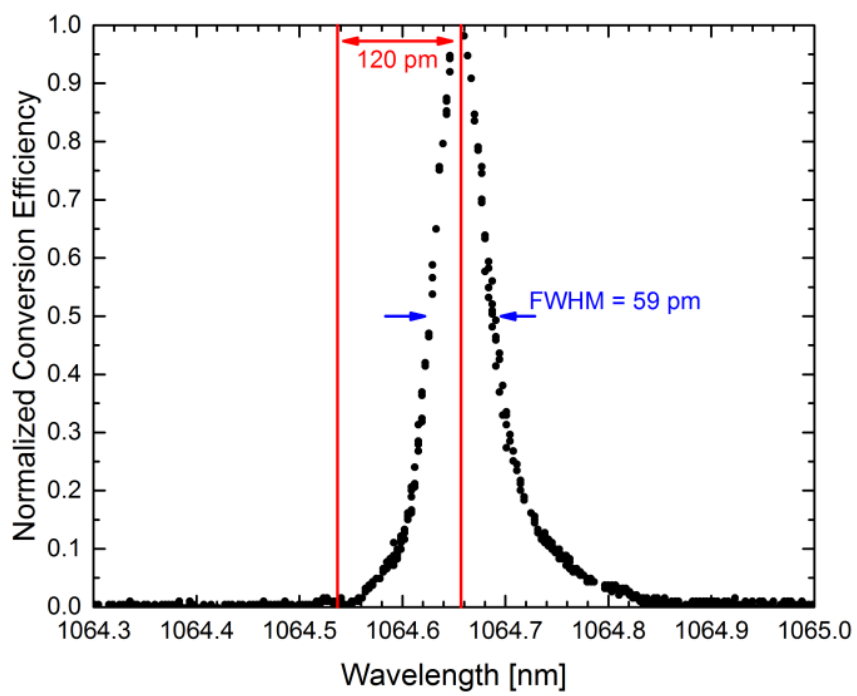

Fig. 4. Measured acceptance bandwidth of the $50 \mathrm{~mm}$ PPLN at a crystal temperature of $61.8^{\circ} \mathrm{C}$. The vertical red lines indicate the two wavelengths used for modulation. A maximum green output power of $180 \mathrm{~mW}$ was generated. The measurement was performed by scanning the temperature of the laser diode.
The SH output power as a function of the NIR input power to the crystal was measured by operating the laser diode at injection currents of $150 \mathrm{~mA}$ to the $\mathrm{MO}$ and $4 \mathrm{~A}$ to the PA, and controlling the NIR power incident on the PPLN by a combination of a PBS and a half-wave plate. The results are shown in Fig. 5. The output follows a quadratic dependence as expected. The maximum SH lightachieved was $166 \mathrm{~mW}$ at an input power of $2.29 \mathrm{~W}$ of NIR light, yielding a conversion efficiency of $3 \% / \mathrm{W}$ which is comparable to former results from similar crystals when using tapered laser diodes[8]. This measurement was also used to calibrate the photodiode for the green light.

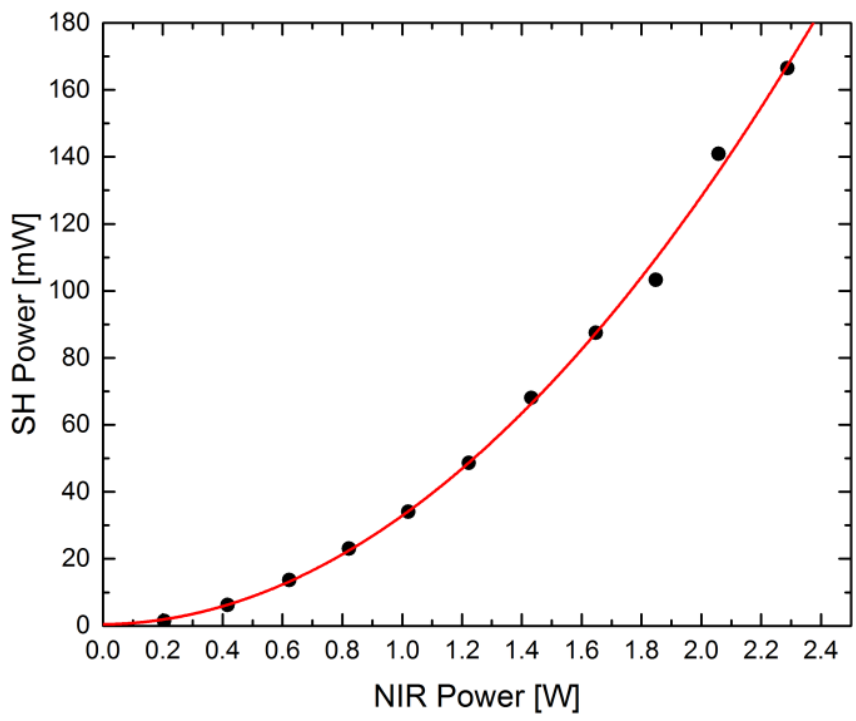

Fig. 5. SH power as a function of the NIR power into the crystal. Black dots are measurements and the red curve is a quadratic fit.

The modulation of the wavelength was performed by connecting a function generator to the modulation input of the current supply for the MO (Arroyo Instruments 4205-DR). All modulation inputs used were square waves with a $50 \%$ duty cycle. When the modulation period is well below the thermal time constants, the temperature of the laser diode tends towards an average temperature which depends on the depth of the current modulation and the duty cycle. For longer modulation periods the temperature of the laser diode can fluctuate during the pulse making the wavelength unstable. A tailored inputpulse can be used to mitigate this effect[14], but the input current pulse will then change shape depending on both the pulse length and duty cycle needed. For the system presented here the wavelength is mostly defined by the current through the DFB section that no such tailoring is needed. To show this the SH light was modulated at $0.1 \mathrm{~Hz}$, see Fig. 5 . Here the timescale of the modulation is significantly slower than all optical, electrical and thermal timescales of the laser diode, including thermal equilibration of the laser mount, meaning it was a quasi-CW case. The average power in the on state for the two pulses was 148.9 $\mathrm{mW}$ and the average off power was $2.74 \mathrm{~mW}$ yielding a modulation depth of $98.2 \%$. The NIR power, on the other hand, only dropped to 2.26 $\mathrm{W}$, corresponding to a $1.3 \%$ decrease in the off state. This result shows that the laser system can be used for pulse on demand applications. 


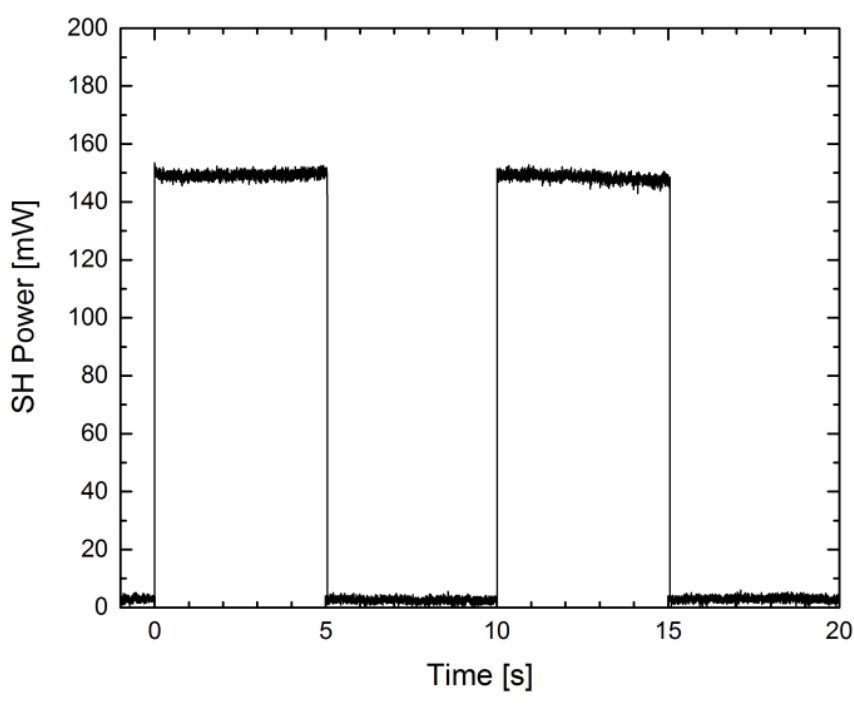

Fig. 6. Modulation of the SH power at $0.1 \mathrm{~Hz}$. A modulation depth of $98.2 \%$ was achieved.

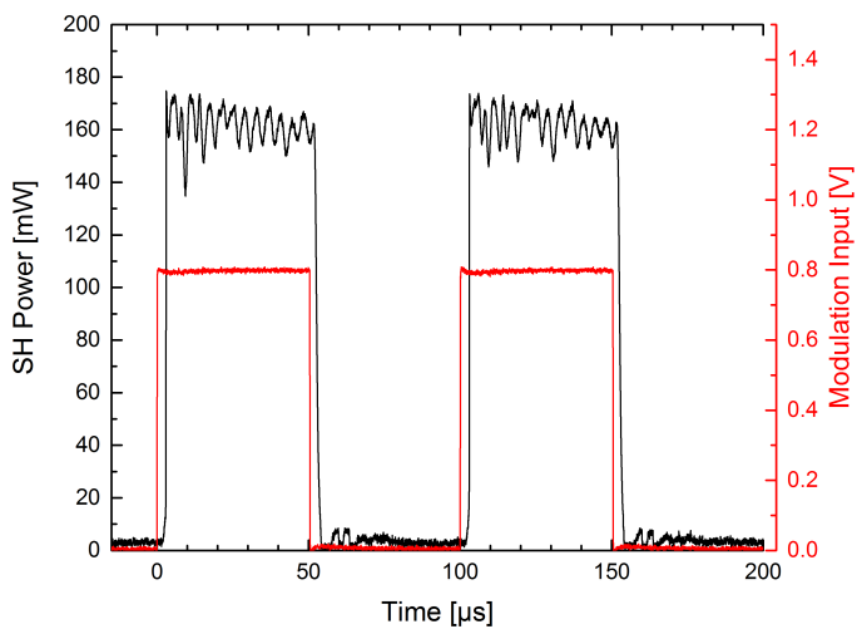

Fig. 7. Modulation of the SH power at $10 \mathrm{kHz}$ (black/left) and the modulation input to the power supply (red/right). A modulation depth of $97.8 \%$ was achieved.

Fig. 6. shows modulation at $10 \mathrm{kHz}$. At this frequency some ringing started to occur in the voltage across the MO. This corresponds well to previously observed limits for the power supply with a modulation of this size, which we believe is why an increased amount of noise is visible in the pulse. The modulation input to the power supply is also shown in the figure to show that the ringing does not originate from the function generator. At $50 \mathrm{kHz}$ the ringing became so pronounced that the pulses could no longer be approximated with a square wave. However, this was only a limitation of the power supply as laser diodes can respond to frequencies in the GHz range[18]. The only parameter changed between figures 5 and 6 is the frequency of the output of the function generator. The average power of the on state in Fig. 6 is $161.7 \mathrm{~mW}$ and for the off state it is $3.50 \mathrm{~mW}$, yielding a modulation depth of $97.8 \%$. For a train of 10 pulses, the pulse to pulse variation of the average power of the on state was below $1 \%$. The slight change in the maximum power between figures 5 and 6 was due to slow temperature fluctuations which were correlated to the room temperature. Very similar results were obtained at $1 \mathrm{~Hz}, 10 \mathrm{~Hz}, 100 \mathrm{~Hz}$ and $1 \mathrm{kHz}$. Since the NIR power is only modulated by $1.5 \%$, the thermal load on the laser is almost constant. This provides the possibility of having pulses on demand, which may be critical for many applications.

\section{Conclusion}

We have demonstrated that the second harmonic output of a singlepass frequency doubled DFB MOPA can be square wave modulated from $\mathrm{CW}$ and up to at least $10 \mathrm{kHz}$ using the method of wavelength detuning by modulating the injection current to the MO. More than $97 \%$ modulation depth was realized by modulating the MO current with a 40 $\mathrm{mA}$ peak to peak amplitude. This corresponds to a wavelength shift of $120 \mathrm{pm}$, and a change of just $1.5 \%$ of the output power of the laser diode itself. The wavelength shift of $120 \mathrm{pm}$ could be achieved with the DFB laser because of the very regular mode hop structure which allowed for operation of the laser diode across a mode hop. Moreover, the low current change needed for wavelength detuning yielded very low thermal fluctuations of the laser diode enabling pulse on demand operation. The upper bound of the modulation frequencies was limited by our electronics. When DFB MOPAs with separate MO and PA electrical contacts become available at higher output power and similar or better mode stability then this scheme can be used to generate arbitrary power modulation with high modulation depth of watt-level visible lasers by low current modulation without any moving parts.

\section{Funding}

This work has been funded by the Innovation Fund Denmark grant number 5016-00076B.

\section{References}

1. K. V Chellappan, E. Erden, and H. Urey, "Laser-based displays: a review.," Appl. Opt. 49(25), F79-98 (2010).

2. L. Chehade, G. Chidlow, J. Wood, and R. J. Casson, "Short-Pulse Duration Retinal Lasers: a review," Clin. Experiment. Ophthalmol. doi: 10.1111/ceo.12754 (2016).

3. J. P. M. Wood, M. Plunkett, V. Previn, G. Chidlow, and R. J. Casson, "Nanosecond pulse lasers for retinal applications," Lasers Surg. Med. 43(6), 499-510 (2011).

4. J. K. Luttrull, C. Sramek, D. Palanker, C. J. Spink, and D. C. Musch, "Long-term safety, high-resolution imaging, and tissue temperature modeling of subvisible diode micropulse photocoagulation for retinovascular macular edema," Retina $\mathbf{X}$, 1-12 (2011).

5. J. Khaydarov, S. Essaian, G. Nemet, A. Shchegrov, N. Simanovskaia, H. Danielyan, G. Gabrielyan, A. Poghosyan, and S Soghomonyan, "Highly-Efficient and Compact Microchip Green Laser Source for Mobile Projectors," Proc. SPIE 7582, 758202 (2010).

6. W. Koechner, "Solid-State Laser Engineering," Springer, (2006).

7. E. Kantola, T. Leinonen, S. Ranta, M. Tavast, and M. Guina, "Highefficiency $20 \mathrm{~W}$ yellow VECSEL," Opt. Express 22(6), 6372-6380 (2014).

8. A. K. Hansen, M. Tawfieq, O. B. Jensen, P. E. Andersen, B. Sumpf, G. Erbert, and P. M. Petersen, "Concept for power scaling second harmonic generation using a cascade of nonlinear crystals," Opt. Express 23(12), 15921 (2015).

9. M. M. Fejer, G. A. Magel, D. H. Jundt, and R. L. Byer, “QuasiPhase-Matched Second Harmonic Generation: Tuning and Tolerances," IEEE J. Quantum Electron. 28(11), 2631-2654 (1992).

10. Y. Kitaoka, T. Yokoyama, K. Mizuuchi, K. Yamamoto, and M. Kato, "Modulated blue second harmonic generation using direct modulation of distributed Bragg reflector laser diode," Electron. Lett. 33(19), 1638-1639 (1997).

11. M. H. Hu, H. K. Nguyen, K. Song, Y. Li, N. J. Visovsky, X. Liu, N. Nishiyama, S. Coleman, L. C. Hughes, J. Gollier, W. Miller, R. Bhat, 
and C. E. Zah, "High-power high-modulation-speed 1060-nm DBR lasers for green-light emission," IEEE Photonics Technol. Lett. 18(4), 616-618 (2006).

12. A. Jechow, M. Schedel, S. Stry, J. Sacher, and R. Menzel, "Highly efficient single-pass frequency doubling of a continuous-wave distributed feedback laser diode using a PPLN waveguide crystal at $488 \mathrm{~nm}$.," Opt. Lett. 32(20), 3035-3037 (2007).

13. D. Jedrzejczyk, R. Güther, K. Paschke, B. Eppich, and G. Erbert, " $200 \mathrm{~mW}$ at $488 \mathrm{~nm}$ From a ppMgO:LN Ridge Waveguide by Frequency Doubling of a Laser Diode Module," IEEE Photonics Technol. Lett. 22(17), 1282-1284 (2010).

14. A. K. Hansen, M. Christensen, D. Noordegraaf, P. Heist, E. Papastathopoulos, O. B. Jensen, and P. M. W. Skovgaard, "Efficient Generation of 1.9 W yellow light by Cascaded Frequency Doubling of a DBR Tapered Diode Laser," Appl. Opt. 55(32), 9270-9274 (2016).

$15 . \quad$ O. B. Jensen, P. E. Andersen, B. Sumpf, K.-H. Hasler, G. Erbert, and $P$. M. Petersen, "High power green light generation by second harmonic generation of single-frequency tapered diode lasers," Proc. SPIE 7582, 758203-1 (2010).

16. "QPC Lasers.," [Online]. Available: http://www.qpclasers.com/products_BLaseSMSE.html. [Accessed: 13-Oct-2016].

$17 . \quad$ O. B. Jensen, P. E. Andersen, B. Sumpf, K.-H. Hasler, G. Erbert, and P. M. Petersen, "1.5 W green light generation by single-pass second harmonic generation of a single-frequency tapered diode laser," Opt. Express 17(8), 6532-6539 (2009).

18. N. Michel, H. Odriozola, C. H. Kwok, M. Ruiz, M. Calligaro, M. Lecomte, O. Parillaud, M. Krakowski, M. Xia, R. V Penty, I. H. White, J. M. G. Tijero, and I. Esquivias, "High modulation efficiency and high power $1060 \mathrm{~nm}$ tapered lasers with separate contacts," Electron. Lett. 45(2), 103-104 (2009). 